DRAFT VERSION NOVEMBER 15, 2018

Preprint typeset using LTEX style emulateapj v. 5/2/11

\title{
IONIZED REFLECTION SPECTRA FROM ACCRETION DISKS ILLUMINATED BY X-RAY PULSARS
}

\author{
D. R. Ballantyne ${ }^{1}$, J. D. Purvis ${ }^{1}$, R. G. Strausbaugh ${ }^{1}$, And R. C. Hickox ${ }^{2}$ \\ Draft version November 15, 2018
}

\begin{abstract}
$\mathrm{X}$-ray reflection signatures are observed around multiple classes of accreting compact objects. Modelling these features yield important constraints on the physics of accretion disks, motivating the development of Xray reflection models appropriate for a variety of systems and illumination conditions. Here, constant density ionized X-ray reflection models are presented for a disk irradiated with a very hard power-law X-ray spectrum $(\Gamma<1)$ and a variable high-energy cutoff. These models are then applied to the Suzaku data of the accreting X-ray pulsar LMC X-4, where very good fits are obtained with a highly ionized reflector responsible for both the broad $\mathrm{Fe} \mathrm{K} \alpha$ line and the soft excess. The ionized reflector shows strong evidence for significant Doppler broadening and is redshifted by $\sim 10^{4} \mathrm{~km} \mathrm{~s}^{-1}$. These features indicate that the reflecting material is associated with the complex dynamics occurring at the inner region of the magnetically-truncated accretion disk. Thus, reflection studies of X-ray pulsar spectra may give important insights into the accretion physics at the magnetospheric radius.

Subject headings: accretion, accretion disks — pulsars: general — pulsars: individual (LMC X-4) — stars: neutron - X-rays: binaries
\end{abstract}

\section{INTRODUCTION}

Many astrophysical systems contain a hard X-ray source in close proximity to dense, relatively cold gas. In many cases the X-rays illuminate the dense gas, which scatters and absorbs the energy before ultimately emitting a spectrum that is imprinted with a variety of diagnostic spectral features, most notably the $\mathrm{Fe} \mathrm{K} \alpha$ fluorescence line (e.g., Lightman \& White 1988; Guilbert \& Rees 1988; George \& Fabian 1991; Matt et al. 1991). Such an X-ray reflection spectrum has been observed from accretion disks around numerous neutron stars (e.g., Cackett et al. 2010) and black holes of all sizes (e.g., Miller 2007). In these systems, modeling the observed X-ray reflection features can lead to important constraints on the ionization stage (Ross et al. 1999; Ross \& Fabian 2005; García \& Kallman 2010), metallicity (Ballantyne et al.2002), and density structure (Ballantyne et al. 2001, 2004, 2005) of the inner accretion disk. In addition, the reflecting region of the disk may be subject to relativistic effects that will sculpt the emission features (e.g., Fabian et al. 1989; Laor 1991) leading to measurements of disk radii and black hole spin (Brenneman \& Reynolds 2006; Miller 2007; Reynolds \& Fabian 2008). It is clear that modeling X-ray reflection features is a very important method in understanding the physics of accreting compact objects; however, such fundamental measurements are only possible by comparing the observed spectra to grids of models that are computed for the appropriate radiative conditions of the system under consideration.

Here, we calculate ionized X-ray reflection models appropriate for accretion powered X-ray pulsars. X-ray pulsars are a class of X-ray binaries in which the neutron star has a strong magnetic field, so that accreting matter follows the field lines and falls into the magnetic poles, generating pulses

\footnotetext{
${ }^{1}$ Center for Relativistic Astrophysics, School of Physics, Georgia Institute of Technology, Atlanta, GA 30332; david.ballantyne@physics.gatech.edu

${ }^{2}$ Department of Physics and Astronomy, Dartmouth College, 6127 Wilder Laboratory, Hanover, NH 03755
}

as the magnetic poles rotate in and out of our line-of-sight (e.g., Nagase 1989; Bildsten et al. 1997). The inner accretion disk is generally believed to be truncated at the magnetospheric or Alfvén radius, where the energy density of the system is dominated by the magnetic field (e.g., Frank et al. 2002). Reflection of hard X-rays from the neutron star by the inner disk has been widely invoked to explain the ubiquitous soft excess component observed in X-ray pulsars (see Hickox et al. 2004 and references therein) as well as fluorescent iron emission lines at $\approx 6.4-6.7 \mathrm{keV}$ (e.g., Choi et al. 1994; Woo et al. 1995; dal Fiume et al. 1998; Burderi et al. 2000; La Barbera et al. 2001; Endo et al. 2002; Naik \& Paul 2003, 2004). Despite the ubiquity of these apparent reflection features in observations of X-ray pulsars, there has been little work aimed at fitting the spectra with a self-consistent, physically-motivated reflection model, which is the goal of the present study. The next section briefly describes the calculation of the grid of reflection models that can be used for $\mathrm{X}$-ray pulsar data, which is then used in Section 3 to fit the Suzaku data of LMC X-4 (Hung et al. 2010). A summary of our conclusions is found in Section 4

\section{CALCULATIONS}

Descriptions of the reflection calculations have been previously published (e.g., Ross \& Fabian 1993; Ross et al. 1999; Ross \& Fabian 2005), so only the main features of the model will be presented here. A one-dimensional, constant density slab of gas with hydrogen number density $n_{\mathrm{H}}$ is illuminated by a X-ray continuum with flux $F_{X}$ defined between $1 \mathrm{eV}$ and $100 \mathrm{keV}$. Thus, for a given spectral shape, models can be described by an ionization parameter

$$
\xi=\frac{4 \pi F_{\mathrm{X}}}{n_{\mathrm{H}}} .
$$

Hydrogen and helium are assumed to be fully ionized (i.e., $\left.n_{e}=1.2 n_{\mathrm{H}}\right)$, and the treated metals $(\mathrm{C}, \mathrm{N}, \mathrm{O}, \mathrm{Mg}, \mathrm{Si}$, and $\mathrm{Fe}$ ) have the abundances of Morrison \& McCammon (1983). The slab has a constant Thomson depth of $\tau_{\mathrm{T}}=15$ ensuring that all X-ray photons interact several times even at the largest 
values of $\xi$. The calculation finishes, and outputs the angleaveraged reflection spectrum, when all 110 zones of the slab reach thermal and ionization balance. The ionization stages of the metals included in the calculation are C V-VII, N VI-VIII, O V-IX, Mg IX-XIII, Si XI-XV and Fe XVI-XXVII.

The continua of $X$-ray pulsars can be described as a hard power-law with a high-energy cutoff:

$$
f_{E} \propto \begin{cases}E^{-\Gamma} & E<E_{\text {cut }} \\ E^{-\Gamma} e^{\left(E_{\text {cut }}-E\right) / E_{\text {fold }}} & E \geq E_{\text {cut }}\end{cases}
$$

where $f_{E}$ is the photon flux per energy interval, $\Gamma$ is the photon index, $E_{\text {cut }}$ is the cutoff energy, and $E_{\text {fold }}$ is the e-folding energy. The cutoff energies for most sources are typically $<30 \mathrm{keV}$ (Hickox et al.2004), much less than the $>100 \mathrm{keV}$ cutoffs assumed in the publicly available power-law reflection models (e.g., Ballantyne et al. 2001; Ross \& Fabian 2005). Similarly, the photon indices of $X$-ray pulsars are $\Gamma \lesssim 1$, significantly harder than the reflection models calculated for fitting AGNs (Ross \& Fabian 2005). These very hard continua also indicate that the reflection spectra will be insensitive to the $1 \mathrm{eV}$ lower limit assumed in the calculation.

A grid of 2376 models is calculated with parameters $5 \mathrm{keV} \leq E_{\text {cut }} \leq 30 \mathrm{keV}$ (in steps of $5 \mathrm{keV}$ ), $5 \mathrm{keV} \leq E_{\text {fold }} \leq$ $30 \mathrm{keV}$ (in steps of $5 \mathrm{keV}$ ), $0.5 \leq \Gamma \leq 1.5$ (in steps of 0.1 ) and $1.5 \leq \log \xi \leq 4.0$ (in steps of $0 . \overline{5}$ ). The ionization parameter is varied by fixing the density at $n_{\mathrm{H}}=10^{19} \mathrm{~cm}^{-3}$ (appropriate for $r \sim 20 r_{g}$ in a radiation pressure dominated accretion disk around a neutron star; $r_{g}=G M / c^{2}$, where $M$ is the mass of a neutron star) and changing $F_{X}$. Two reflection spectra with different $\xi$ are shown in Figure 1 The features in this spectra are qualitatively very similar to those used in modeling observations of AGNs. However, because of the high density in neutron star disks and the very hard illuminating power-law, the $\log \xi=3$ model predicts a strong bremsstrahlung dominated soft excess at energies $\lesssim 3 \mathrm{keV}$ from the hot surface of the X-ray heated disk. The thermal bremsstrahlung continuum is modified by Comptonization in the hot atmosphere so that it approximately resembles the blackbody spectrum typically used to fit the observed soft excess. In addition, the low ionization model predicts several strong recombination lines from ionized $\mathrm{C}, \mathrm{N}$ and $\mathrm{O}$ in the slab which, if measured, could constrain the abundances of the accreting gas. Interestingly, the soft excesses in X-ray pulsar spectra are observed to be featureless in observations with CCD resolution (Hickox et al. 2004); therefore, if X-ray reflection is responsible for the soft excess, the ionization parameter of the reflector must be large.

\section{APPLICATION TO LMC X-4}

To determine if the ionized reflection models provide a good description of the soft excesses observed from X-ray pulsars, the grid of models described above is fit to three phase-averaged Suzaku spectra of LMC X-4 (Hunget al. 2010). LMC X-4 is a high-mass X-ray binary system consisting of a $1.29 M_{\odot}$ neutron star accreting from a 14.5 $M_{\odot}$ O8 III companion (Kelley et al. 1983; van der Meer et al. 2007; Rawls et al. 2011). The neutron star rotates with a

\footnotetext{
3 The exact value of the density at the surface of a X-ray pulsar accretion disk is unknown (and will likely be functions of the disk radius and accretion rate; e.g., Shakura \& Sunvaev 1973), but is expected to be $n_{\mathrm{H}} \gg 10^{20} \mathrm{~cm}^{-3}$, a region where calculations become computationally prohibitive. Recall, however, that the $\alpha$-disk model gives the midplane or an average density, and the density at the surface is likely to be much lower.
}

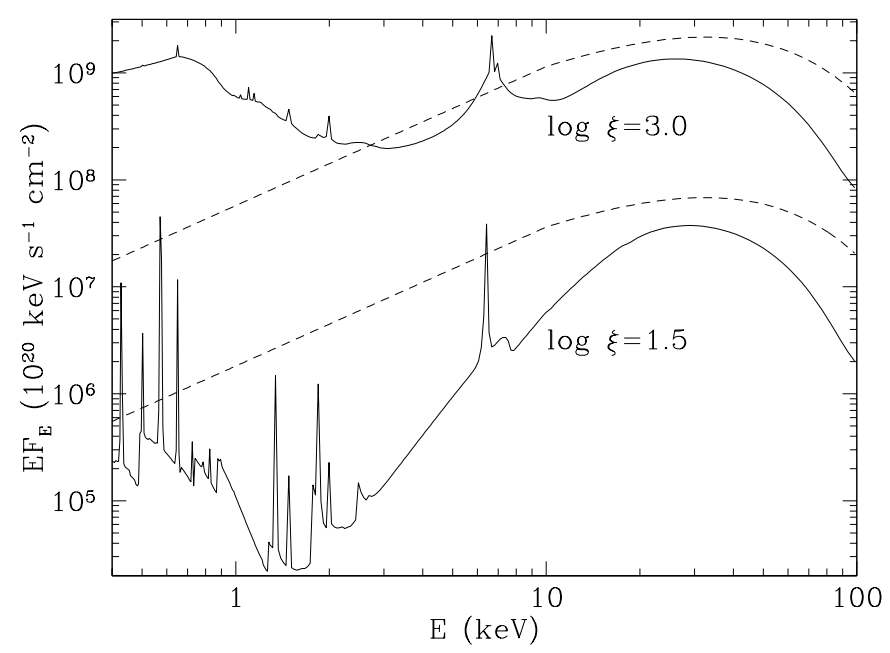

Figure 1. Reflection spectra from a constant density slab irradiated by a power-law with a high-energy cutoff (Eq. 2) with $\Gamma=0.7, E_{\text {cut }}=10 \mathrm{keV}$ and $E_{\text {fold }}=25 \mathrm{keV}$ (dashed lines). The solid lines plot the reflection spectra for both a weakly ionized slab $(\log \xi=1.5)$ and a strongly ionized one $(\log \xi=3.0)$. The highly irradiated slab produces a strong bremsstrahlung dominated soft excess at energies $\lesssim 3 \mathrm{keV}$.

period of $\sim 13.5 \mathrm{~s}$, orbits its companion with a period of $\sim 1.4$ days (White 1978), and exhibits a long-term periodicity of $30.5 \pm 0.5$ days that is believed to be caused by a precessing accretion disk that periodically obscures the neutron star (e.g., Lang et al. 1981; Heemskerk \& van Paradijs 1989; Naik \& Paul 2003).

LMC X-4 was observed by the Suzaku X-ray observatory in 2008 January-April, with exposures of $\approx 20 \mathrm{ks}$ duration taken at three different phases in the superorbital cycle $\left(\phi_{30}=0.39\right.$, 0.27 , and 0.07 for Observations 1, 2, and 3, respectively). Details of the data reduction and spectral extraction are presented in Hung et al. (2010). Phase-averaged spectra were extracted in the $0.5-10 \mathrm{keV}$ energy range for the X-ray Imaging Spectrometer (XIS) CCD detector, and the 10-50 keV range for the PIN instrument of the Hard X-ray Detector (HXD), and the spectra fitted jointly for each observation. Hung et al. (2010) found that the broad-band Suzaku spectra are well described by a hard power-law with a high-energy cutoff, a soft $\mathrm{X}$-ray excess, and a strong iron emission line, similar to previous studies (e.g., Woo et al. 1996; La Barbera et al. 2001; Naik \& Paul 2003). The best-fit models also included emission features at $\approx 0.6 \mathrm{keV}$ and $\approx 1 \mathrm{keV}$ that were identified by Hung et al. (2010) as emission from highly ionized $\mathrm{O}$ and $\mathrm{Ne}$, respectively. An analysis of the energy-resolved pulse profiles showed that the phase offset between hard $(>2 \mathrm{keV})$ and soft $(<1 \mathrm{keV})$ pulsations varies with time, as has been observed for the similar X-ray pulsars Her X-1 (Ramsay et al. 2002; Zane et al. 2004) and SMC X-1 (Neilsen et al. 2004; Hickox \& Vrtilek 2005). As discussed in Hung et al. (2010), this behavior is consistent with the origin of the soft component as the illuminated inner region of the precessing accretion disk.

In this paper we further explore the physical origin of the reflected emission in LMC X-4, by re-analyzing the Suzaku spectra using our reflection model. XSPEC v.12.7 Arnaud 1996) is used to fit the Suzaku data. Errorbars on the best fit parameters are the $2 \sigma$ uncertainties for one parameter of interest (i.e., $\Delta \chi^{2}=2.71$ ).

\subsection{Spectral Fits}


Table 1

LMC X-4 Spectral Fitting Results

\begin{tabular}{cccc}
\hline \hline Parameter & Observation 1 & Observation 2 & Observation 3 \\
\hline$\chi_{\nu}^{2}(385$ d.o.f. $)$ & 1.13 & 1.22 & 1.04 \\
$N_{\mathrm{H}}\left(\times 10^{22} \mathrm{~cm}^{-2}\right)($ fixed $)$ & 0.057 & 0.057 & 0.057 \\
$F_{0.6-50 \mathrm{keV}\left(\mathrm{erg} \mathrm{cm}^{-2} \mathrm{~s}^{-1}\right)}$ & $8.6 \times 10^{-10}$ & $1.4 \times 10^{-9}$ & $1.1 \times 10^{-9}$ \\
\hline \multicolumn{4}{c}{ Continuum } \\
\hline$\Gamma$ & $0.68_{-0.02}^{+0.01}$ & $0.67 \pm 0.01$ & $0.67 \pm 0.02$ \\
$E_{\text {cut }}(\mathrm{keV})$ & $18.7 \pm 0.5$ & $18.0 \pm 0.4$ & $17.9_{-0.7}^{+0.6}$ \\
$E_{\text {fold }}(\mathrm{keV})$ & $13.3 \pm 0.5$ & $15.6_{-0.4}^{+0.5}$ & $14.9 \pm 0.7$ \\
\hline Redshifted \& Doppler Broadened Ionized Reflection Spectrum \\
\hline $\log \xi$ & $3.02 \pm 0.01$ & $2.99 \pm 0.01$ & $3.02 \pm 0.01$ \\
$z$ & $0.034_{-0.003}^{+0.011}$ & $0.034_{-0.009}^{+0.004}$ & $0.036_{-0.021}^{+0.012}$ \\
$\sigma_{6} \mathrm{keV}(\mathrm{keV})$ & $0.19_{-0.05}^{+0.07}$ & $0.18_{-0.05}^{+0.06}$ & $0.37_{-0.21}^{+0.16}$
\end{tabular}

Unblurred Reflection Spectrum

\begin{tabular}{cccc}
\hline $\log \xi$ (fixed) & 1.5 & 1.5 & 1.5 \\
\hline \multicolumn{4}{c}{ Gaussian Emission Component } \\
\hline$E(\mathrm{keV})$ & $0.94 \pm 0.01$ & $0.95 \pm 0.01$ & $0.94 \pm 0.01$ \\
$\sigma(\mathrm{keV})$ & $0.11 \pm 0.01$ & $0.10 \pm 0.01$ & $0.11 \pm 0.01$ \\
$\mathrm{EW}(\mathrm{eV})$ & 129 & 107 & 99 \\
\hline
\end{tabular}

\begin{tabular}{cccc}
\hline \multicolumn{5}{c}{ Relative Normalizations } \\
\hline$A_{\text {XIS1 }} 1.01 \pm 0.01$ & $0.95 \pm 0.01$ & $1.01 \pm 0.01$ \\
$A_{\text {PIN }}$ & $1.09_{-0.03}^{+0.02}$ & $1.03 \pm 0.01$ & $1.06_{-0.02}^{+0.03}$ \\
\hline \multicolumn{5}{c}{$0.6-50 \mathrm{keV}$ Flux Ratios } \\
\hline Ionized Reflector/Power-Law & 0.17 & 0.09 & 0.11 \\
Neutral Reflector/Power-Law & 0.05 & 0.03 & 0.04
\end{tabular}

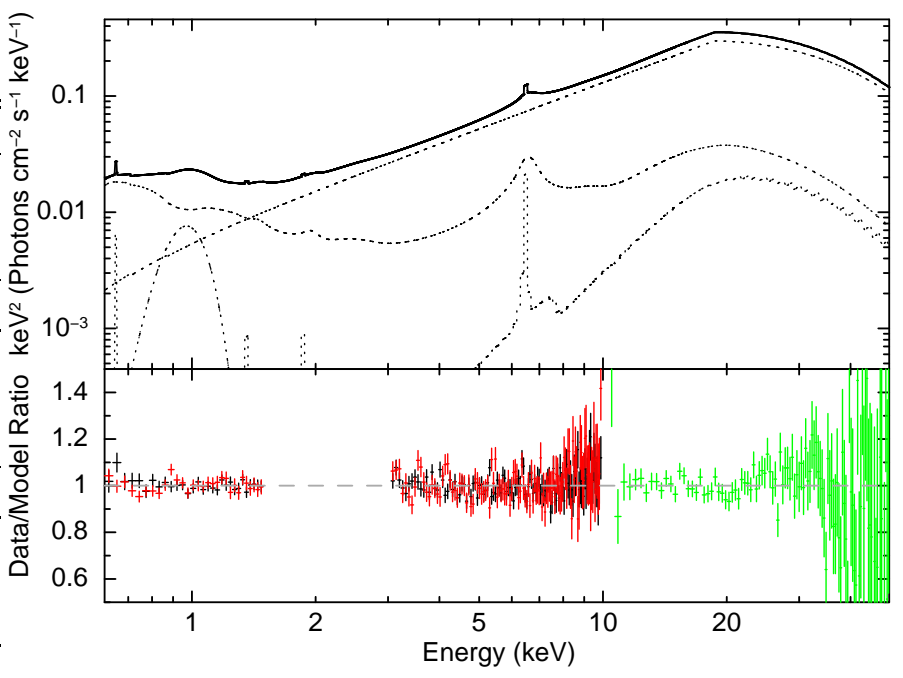

Figure 2. The best-fitting model (top panel) and data-to-model ratio (lower panel) found for Observation 1 of LMC X-4. In the upper panel, the thick solid line plots the total model, while the individual components (the continuum, the reflectors and the Gaussian emission line) are indicated with dotted lines. In the bottom panel, the black/red/green data points are from the Suzaku/FI/XIS1/PIN detectors, respectively.

can be described by a redshifted and Doppler broadened ionized $\mathrm{Fe} \mathrm{K} \alpha$ line from the same reflector. The width of the ionized Fe $\mathrm{K} \alpha$ line $(\sim 0.2 \mathrm{keV})$ is less than the one determined by Hung et al. (2010) $(\sim 0.4 \mathrm{keV})$ because the line is naturally broadened due to Comptonization (see Figure 1). The low-ionization reflector self-consistently explains the narrow $\mathrm{O}$ VIII and Fe $\mathrm{K} \alpha$ lines (Hung et al. 2010). However, the reflection components are a small fraction of the total observed

Note. - The Doppler broadening was modeled with the 'gsmooth' convolution model in XSPEC with the Gaussian width $\sigma(E)=\sigma_{6} \mathrm{keV}(E / 6 \mathrm{keV})$. flux. The low- $\xi$ reflector contributes only $\approx 4 \%$ of the total flux for each observation, indicating that this emission region subtends a consistently small solid angle as seen from the pulsar, and therefore originates from the outer disk, the accretion stream, or the companion star. In contrast, the highly ion-

The spectral model used in the fitting is the same as the one used by Hung et al. (2010) except the blackbody and emission lines are replaced by the ionized reflection model. Hung et al. (2010) found that both a narrow and broad $\mathrm{Fe} \mathrm{K} \alpha$ line were required to fit the Suzaku data. Therefore, two reflectors are used in the new spectral model, with one subject to Doppler broadening using 'gsmooth'. The two reflection components and the primary power-law all have the same $\Gamma, E_{\text {cut }}$ and $E_{\text {fold. }}$. The ionization parameter of the unbroadened reflection spectrum is fixed at $\log \xi=1.5$ so that it can account for the observed narrow, neutral $\mathrm{Fe} \mathrm{K} \alpha$ line, but its normalization is allowed to vary. The results of all three fits are shown in Table 1 and show that in every case the ionized reflection model is an excellent description of the broadband spectrum of LMC X-4 (see Figure 2). In order to fit the centroid of the broad Fe $\mathrm{K} \alpha$ line at $\sim 6.5 \mathrm{keV}$ (Hung et al. 2010), the ionized reflection spectrum (which produces a $6.7 \mathrm{keV}$ line from $\mathrm{He}$ like iron) must be redshifted by $\sim 10^{4} \mathrm{~km} \mathrm{~s}^{-1}$. Interestingly, the fits require an additional Gaussian line with an equivalent width $(\mathrm{EW})$ of $\sim 100 \mathrm{eV}$ at an energy of $\approx 0.94 \mathrm{keV}$. The possible origin of this line is discussed below.

As seen in Figure 2, and in agreement with the expectations of Hickox et al. (2004), the soft excess of LMC X-4 is almost entirely explained by a highly ionized reflection spectrum, and is produced by the thermal bremsstrahlung emitted by the hot, ionized surface of the irradiated region. In addition, the broadened Fe $\mathrm{K} \alpha$ line required by Hung et al. (2010) this increases to $17 \%$ for Observation 1. As these observations were taken at different super-orbital periods, as the inner accretion disk precess around the pulsar, then this changing fraction strongly implies that the ionized reflector arises from the inner regions of the magnetically-truncated accretion disk, consistent with the origin of the broadened Fe $\mathrm{K} \alpha$ line. Moreover, the change in flux ratio indicates that our view of the inner reflecting region evolves with the super-orbital period, as expected for a warped precessing disk (Hung et al. 2010).

The fits require an additional, broad emission component between 0.9 and $1 \mathrm{keV}$ that does not appear in the reflection model (the best fit without this feature results in a $\Delta \chi^{2} \approx$ +200 ). This emission is well modeled by a broad Gaussian component with a centroid energy of $0.94 \mathrm{keV}, \sigma=0.1 \mathrm{keV}$, and $\mathrm{EW} \approx 100 \mathrm{eV}$. These properties are significantly different than the weak, narrow line that Hung et al. (2010) needed at a similar energy and tentatively identified as arising from highly ionized $\mathrm{Ne}$. In this case, a narrow emission line is strongly rejected by the fit, and thus favors an interpretation that the Gaussian component is modeling a smooth part of the continuum that is missing from the highly ionized reflection spectrum. The energy and width of this feature are very similar to a Gaussian bump in the XMM-Newton RGS spectra of LMC X-4 analyzed by Neilsen et al. (2009), who modeled the soft excess using a variety of different continua and persis- 
tently observed an additional Gaussian component. Thus, this feature is unlikely to be an instrumental artifact, but a true unmodeled component of the continuum (a similar feature was also found in the spectrum of Her X-1; Jimenez-Garate et al. 2002). The energy range spanned by this emission feature encompasses many transitions of Fe XVII-XXIII (e.g., Brown et al. 2002), which, for this value of $\xi$, are expected to be weak. However, there will likely be a steep ionization gradient on the surface of the inner accretion disk that is being parameterized here by only a single ionization parameter. In this scenario, a single ionized reflection spectrum may not be able to account for all the spectral complexity at $\lesssim 1 \mathrm{keV}$, a region which is very sensitive to the ionization parameter (e.g., García \& Kallman 2010). Whatever the origin of this feature, it only contributes $9 \%$ of the soft excess flux (measured between 0.6 and $2 \mathrm{keV}$ ), as compared to $56 \%$ produced by the ionized reflector. Therefore, this unmodeled emission does not alter the conclusion that the highly ionized reflector provides a good description of the soft excess in LMC X-4.

The redshift and Doppler broadening of the ionized reflecting gas indicates that it is subject to strong dynamical effects. One possibility is relativistic smearing from motions close to the neutron star (e.g., Fabian et al. 1989). However, tests with the 'rdblur' model show that good fits can only be obtained if the inner radius of the reflector was $\sim 20-40 r_{g}$ from the star. Identifying this radius with the magnetospheric radius implies a magnetic field strength of $\sim 10^{9} \mathrm{G}$ (Ghosh \& Lamb 1978, 1979; Frank et al. 2002), significantly smaller than the $\sim 10^{13} \mathrm{G}$ field strength required to explain the stability of the LMC X-4 pulse period (Woo et al. 1996). Moreover, if the disk extended down to $\sim 20-40 r_{g}$ the accretion luminosity from the disk would overwhelm the observed spectrum unless the accretion rate was $<0.07$ of the Eddington rate, which is problematic given the observed luminosity of $3 \times 10^{38} \mathrm{erg} \mathrm{s}^{-1}$. Thus, as indicated by its low covering factor, this gas must originate much farther out from the neutron star, perhaps from the inner edge of the disk as it interacts with the magnetosphere. With a magnetic field strength of $\sim 10^{13} \mathrm{G}$, the magnetospheric radius of LMC X-4 is $\sim 2 \times 10^{8} \mathrm{~cm}$ which would have an orbital velocity of $\sim 10^{4} \mathrm{~km} \mathrm{~s}^{-1}$ (Hung et al. 2010), in good agreement with both the measured redshift and the velocity width at $6.7 \mathrm{keV}$. This conclusion is supported by the results of Neilsen et al. (2009) who uncovered evidence in high resolution gratings spectra for multiple regions of high-speed ionized gas flows. It is clear that ionized reflection spectra not only explain the soft-excess of X-ray pulsars, but allows a probe of the complicated dynamics associated with the interaction of the magnetosphere and accretion disk.

\section{CONCLUSIONS}

This Letter presents new reflection models developed for accreting X-ray pulsars. Thermal bremsstrahlung subject to Comptonization from an ionized X-ray heated disk is found to be a natural explanation for the soft excess observed in the spectra of LMC X-4, and, presumably, other X-ray pulsars which exhibit Fe $\mathrm{K} \alpha$ lines and soft excesses. Fitting the Suzaku data of LMC X-4 nicely illustrates the potential of reflection modeling for elucidating the physics of these systems. We found that the observed broad $\mathrm{Fe} \mathrm{K} \alpha$ line could be explained as originating from a redshifted $\left(\sim 10^{4} \mathrm{~km} \mathrm{~s}^{-1}\right)$ highly ionized reflector subject to Doppler broadening. These velocities are consistent with those expected from the magnetospheric radius of $\sim 2 \times 10^{8} \mathrm{~cm}$ (Hung et al. 2010). Thus, applying this grid of reflection models to both archival and future X-ray pulsar spectra will provide important constraints on the ionization state, metallicity and dynamics at the inner edge of magnetically-truncated accretion disks.

The authors thank the anonymous referee for a very helpful report. This work was supported in part by NSF award AST 1008067 to DRB. RCH acknowledges Suzaku grant NNX08AI17G.

\section{REFERENCES}

Arnaud K.A., 1996, in Jacoby G., Barnes J., eds, Astronomical Data Analysis Software and Systems V, ASP Conf. Ser. Vol. 101, 17 Ballantyne, D.R., Ross, R.R. \& Fabian, A.C., 2001, MNRAS, 327, 10 Ballantyne D.R., Fabian A.C., Ross R.R., 2002, MNRAS, 329, L67 Ballantyne D.R., Turner N.J., Blaes O.M., 2004, ApJ, 603, 436 Ballantyne, D.R., Turner, N.J. \& Young, A.J., 2005, ApJ, 619, 1028 Bildsten, L., Chakrabarty, D., Chiu, et al., 1997, ApJS, 113, 367 Brenneman, L.W. \& Reynolds, C.S., 2006, ApJ, 652, 1028

Brown, G.V., Beiersdorfer, P, Liedahl, D.A. et al., 2002, ApJS, 140, 589

Burderi, L., Di Salvo, T., Robba, N.R., et al., 2000, ApJ, 530, 429 Cackett, E.M., Miller, J.M., Ballantyne, D.R., et al., 2010, ApJ, 720, 205

Choi, C.S., Nagase, F., Makino, F., et al., 1994, ApJ, 437, 449

dal Fiume, D., Orlandini, M., Cusumano, G., et al., 1998, A\&A, 329, L41

Endo, T., Ishida, M., Masai, K., et al., 2002, ApJ, 574, 879

Fabian, A.C., Rees, M.J., Stella, L. \& White, N.E., 1989, MNRAS, 238, 729

Frank, J., King, A. \& Raine, D.J., 2002, in Accretion Power in Astrophysics (3rd ed.; Cambridge; Cambridge Univ. Press)

García J. \& Kallman T.R., 2010, ApJ, 718, 695

George I.M., Fabian A.C., 1991, MNRAS, 249, 352

Ghosh, P. \& Lamb, F.K., 1978, ApJ, 223, L83

Ghosh, P. \& Lamb, F.K., 1979, ApJ, 232, 259

Guilbert P.W., Rees M.J., 1988, MNRAS, 233, 475

Heemskerk, M. H. M. \& van Paradijs, J. 1989, A\&A, 223, 154

Hickox, R.C., Narayan, R. \& Kallman, T.R., 2004, ApJ, 614, 881

Hickox, R. C. \& Vrtilek, S. D. 2005, ApJ, 633, 1064

Hung, L.-W., Hickox, R.C., Boroson, B.S. \& Vrtilek, S.D., 2010, ApJ, 720, 1202

Jimenez-Garate, M.A., Hailey, C.J., den Herder, J.W., Zane, S. \& Ramsay, G., 2002, ApJ, 578, 391

Kelley, R. L., Jernigan, J. G., Levine, A., Petro, L. D., \& Rappaport, S. 1983, ApJ, 264, 568

La Barbera, A., Burderi, L., Di Salvo, T., et al., 2001, ApJ, 553, 375

Lang, F. L., et al. 1981, ApJ, 246, L21

Laor, A., 1991, ApJ, 376, 90

Lightman A.P., White T.R., 1988, ApJ, 335, 57

Matt G., Perola G.C., Piro L., 1991, A\&A, 247, 25

Miller, J.M., 2007, ARA\&A, 45, 441

Morrison, R. \& McCammon, D., 1983, ApJ, 270, 119

Nagase, F. 1989, PASJ, 41, 1

Naik, S. \& Paul, B. 2003, A\&A, 401, 265

-. 2004, ApJ, 600, 351

Neilsen, J., Hickox, R. C., \& Vrtilek, S. D. 2004, ApJ, 616, L135

Neilson, J., Lee, J.C., Nowak, M.A., Dennerl, K. \& Vrtilek, S.D., 2009, ApJ, 696, 182

Ramsay, G., Zane, S., Jimenez-Garate, M. A., et al., 2002, MNRAS, 337, 1185

Rawls, M.L., Orosz, J.A., McClintock, J.E. et al., 2011, ApJ, 730, 25

Reynolds, C.S. \& Fabian, A.C., 2008, ApJ, 675, 1048

Ross R.R., Fabian A.C., Young A.J., 1999, MNRAS, 306, 461

Ross, R.R. \& Fabian, A.C., 1993, MNRAS, 261, 74

Ross, R.R. \& Fabian, A.C., 2005, MNRAS, 358, 211

Shakura N.I., Sunyaev R.A., 1973, A\&A, 24, 337

van der Meer, A., Kaper, L., van Kerkwijk, M. H., et al., 2007, A\&A, 473, 523

White, N. E. 1978, Nature, 271, 38

Woo, J. W., Clark, G. W., Blondin, J. M., et al., 1995, ApJ, 445, 896

Woo, J.W., Clark, G.W., Levine, A.M., Corbet, R.H.D., \& Nagase, F., 1996, ApJ, 467, 811

Zane, S., Ramsay, G., Jimenez-Garate, M. A., et al., 2004, MNRAS, 350, 506 